\title{
Optogenetics: a futuristic panacea in genetics
}

\author{
Dito Anurogo', Taruna Ikrar ${ }^{2,3,4}$ \\ ${ }^{1}$ Biomedical Sciences Master Program, Faculty of Medicine, Universitas Gadjah \\ Mada, Yogyakarta, Indonesia, ${ }^{2}$ School of Medicine, University of California, Irvine, \\ USA, ${ }^{3}$ Brain Circulation Institute of Indonesia (BCII), Surya University, Indonesia, \\ ${ }^{4}$ Faculty of Medicine, University Hasanuddin, Makassar, Indonesia
}

DOI: http://dx.doi.org/10.19106/JMedScieSup004804201604

\section{ABSTRACT}

Optogenetics is a constellation of optics, genetics, and bioengineering which unites genetic engineering with optics to determine and manage the function of genetically-targeted groups of cells with light, often in the intact animal, via lightsensitive microbial membrane proteins (opsins). Light-sensitive genes, especially the genetically targeted light-gated channels channelrhodopsin-2 (ChR2) and halorhodopsin (NpHR), results in intracellular ion flow during optical illumination. Afterwards, the neurons encounter a series of changes resulting from membrane depolarization or hyperpolarization. Despite its neuroscience roots, optogenetics could potentially be applied in neuropsychocardioncology (neurology, psychiatry, oncology, and cardiology). This critical review will explicate a comprehensive summary of the roles of optogenetics in the field of neuropsychocardioncology. Optogenetics can be potentially developed as neuroprosthetics and direct NpHR in the management of spastic movement disorders. Optogenetics can help controlling laryngeal muscles contractions in vivo, using both transgenic ChR2expressing mice and viral transduction of muscle. The efficacy of optogenetics has been proven in epilepsy. Transduction of pyramidal cells in the cortex with halorhodopsin and photoinhibition of the neurons were found to decrease electrical seizure activity. Optogenetics and DREADD technologies are in their early stages, particularly with respect to PD research or therapy. Behavioral deficits in autism and schizophrenia might arise from the elevation of cellular balance during excitation/inhibition ( $\mathrm{E} / \mathrm{l}$ balance) within neuronal microcircuits. This hypothesis was tested by optogenetically elevating the $\mathrm{E} / \mathrm{l}$ balance in the medial-prefrontal cortex using a step-function opsin (SSFO), as well as redshifted opsins (C1V1). Increased excitation in excitatory pyramidal neurons leads to social-cognitive dysfunction, which are similar to those seen in autism. Cortical gamma oscillations are also an indicator of enhanced information processing, which is highly affected in schizophrenic patients. Using optogenetic technology, researchers were able to characterize the impacts of phosphatidylinositol 3-kinase 
(PI3K) in Rac1-dependent lamellipodial motility in PC-3 prostate cancer cells. With its downstream action on Rac1, PI3K has an important role in the initiation of lamellipodial extension, which contributes to prostate cancer cell invasion and metastasis. As in Parkinson's disease, human cells can be engineered to deliver the excitatory (hM3Dq receptor) and/or the inhibitory (hM4Di receptor) signals to regulate the extent of cellular activity. The optogenetic TCU (tandem-cellunit) strategy could be valuable in assessing grafted tissue integration and cell delivery in the myocardium during cardiac tissue repair procedures. Low-energy pacing strategies could be analyzed by optogenetic investigations. In addition, optical stimulation could also be utilized to assess the strategic structures of the conduction system. Optogenetic studies have contributed to a better understanding of the neural circuits affected in many disorders. A conceptual and mutual understanding of multidisciplinary approaches and collaborations would enable researchers, clinicians, stakeholders, and the government to develop and apply optogenetics in comprehensive medical services and health care.

Keywords: optogenetics; opsins; neuropsychocardioncology; SSFO; DREADD; TCU 